\title{
EFECTO DE INDUCTORES DE RESISTENCIA SOBRE LAS SIGATOKAS NEGRA (Mycosphaerella fijiensis MORELET) Y AMARILLA (Mycosphaerella musicola LEACH) EN PLÁTANO
}

\section{EFFECT OF QUEMICAL INDUCERS OF DISEASE RESISTANCE TO BLACK (Mycosphaerella fijiensis MORELET) AND YELLOW SIGATOKAS (Mycosphaerella musicola LEACH) IN PLANTAIN}

\author{
Carolina Ramírez ${ }^{1}$, Jairo Castaño ${ }^{2}$, Bernardo Villegas ${ }^{3}$, Manuel Aristizábal $^{4}$
}

\begin{abstract}
${ }^{1}$ I.A., klarl2000@gmail.com; ${ }^{2}$ I.A., Ph.D. Facultad de Ciencias Agropecuarias, Universidad de Caldas, Calle 65 №26-10, Manizales, Caldas, Colombia, Autor para correspondencia: jairo.castano_z@ucaldas.edu.co; ${ }^{3}$ I.A. M.Sc. Facultad de Ciencias Agropecuarias, Universidad de Caldas, bernardo.villegas@ucaldas.edu.co; ${ }^{4}$ I.A. M.Sc. Facultad de Ciencias Agropecuarias, Universidad de Caldas, manuel.aristizabal@ucaldas.edu.co
\end{abstract}

Rev. U.D.C.A Act. \& Div. Cient. 15(12): 363 - 371, 2012

\section{RESUMEN}

Se evaluó el efecto individual de los inductores de resistencia acibenzolar-s-metil y fosfito de potasio y en mezcla con propiconazole sobre la severidad de las Sigatokas negra y amarilla y el crecimiento y producción del plátano Dominico Hartón (Musa AAB). Además, se determinó el impacto económico de los tratamientos. El estudio, se llevó a cabo en la granja Montelindo de la Universidad de Caldas, con precipitación anual de $2100 \mathrm{~mm}$, humedad relativa promedio del $76 \%$, temperatura promedio de $23,5^{\circ} \mathrm{C}$ y altitud de $1010 \mathrm{~m}$. Los tratamientos generaron una situación fitosanitaria similar al manejo convencional. En términos de rendimiento, los tratamientos en los que se usó el inductor acibenzolar-s-metil en sus dos dosis ( 40 y $80 \mathrm{~mL} \mathrm{ha}^{-1}$ ) y en mezcla con el fungicida propiconazole ( 40 y $200 \mathrm{~mL} \mathrm{ha}^{-1}$, respectivamente), tuvieron diferencias de 6,0, 3,0 y 5,4t ha ${ }^{-1}$, respectivamente, con respecto al testigo no tratado; los tratamientos con fosfito de potasio y el fungicida solamente, no presentaron diferencia en cuanto a rendimiento, en comparación con el testigo.

Palabras clave: Severidad, rendimiento, acibenzolar-s-metil, fosfito de potasio.

\section{SUMMARY}

The individual effect of resistance inducers acibenzolar-smethyl and potassium phosphate and the mixture with propi- conazole on the severity of black and yellow Sigatokas as well as on growth and production of Dominico Harton plantain (Musa AAB) was evaluated. Also the economic impact of the treatments was determined. The study was carried out at the 'Montelindo' farm (property of the Universidad de Caldas) with an annual rainfall of $2100 \mathrm{~mm}$, relative humidity of $76 \%$, average temperature of $23.5^{\circ} \mathrm{C}$ and altitude of $1010 \mathrm{~m}$. Treatments caused a phytosanitary situation, similar to the conventional management; in terms of yield, those treatments with the two doses of acibenzolar-s-methyl $\left(40\right.$ y $\left.80 \mathrm{~mL} \mathrm{ha}^{-1}\right)$ and in mixture with the fungicide propiconazole 40 y $200 \mathrm{~mL}$ $\mathrm{ha}^{-1}$, showed differences of 6.0, 3.0 and 5.4tha ${ }^{-1}$ respectively, with respect to the untreated control; treatments with potassium phosphite and the fungicide alone did not show statistical difference with the control.

Key words: Severity, yield, acibenzolar-s-methyl, potassium phosphite.

\section{INTRODUCCIÓN}

En 2010, Colombia participó con el 7,9\% de la producción mundial de plátano y ocupó el tercer lugar entre los países con mayor producción, después de Uganda y de Ghana (FAOSTAT, 2012). El país presenta una ventaja comparativa en el rendimiento del cultivo, en el ámbito mundial, con 8,5t $\mathrm{ha}^{-1}$. Los departamentos de Antioquia, de Quindío, de Arauca, de Caldas, de Meta, del Valle, de Risaralda, del Tolima, de Nariño y del Cauca, en su orden, son los mayores pro- 
ductores y representan el $72 \%$ de la producción y el $65 \%$ del área sembrada, que para el 2010 fue de 368.392ha (Agronet, 2012).

Aunque varias especies de Mycosphaerella pueden estar involucradas en el complejo de Sigatokas del plátano (Arzanlou et al. 2008), las más destructivas, en la esfera nacional y mundial, son la Sigatoka negra, causada por Mycosphaerella fijiensis Morelet, y la Sigatoka amarilla, causada por Mycosphaerella musicola Leach; estos hongos atacan las hojas y provocan pérdidas de más del $50 \%$ en el rendimiento, ya que al no ser tratadas a tiempo el área foliar, fotosintéticamente activa, se reduce de manera considerable (Craenen \& Ortiz, 2003).

En las plantaciones comerciales destinadas a la producción de banano y de plátano para exportación, se requieren entre 35 - 50 aspersiones anuales de fungicidas, para el control de las Sigatokas negra y amarilla (Chica et al. 2004). Debido a la acción nociva de los agroquímicos sobre el ambiente y la población rural, ha sido necesario desarrollar sistemas de producción con tecnologías limpias, es decir, con mínima aplicación de agroquímicos. Esto, a su vez, constituye un reto para los investigadores en búsqueda de esquemas más completos para el manejo integrado de estas enfermedades, que incluyan, por ejemplo, el empleo de inductores de resistencia (Patiño, 2002), que no muestran actividad micotóxica, pero, en unos casos, pueden tener acción de protección local inducida y, en otros, acción sistémica. Pueden ser de amplio espectro, puesto que pueden proteger las plantas contra enfermedades causadas por diversos patógenos, como hongos, bacterias, fitoplasmas, virus y nematodos (Riveros, 2001).

La inducción de resistencia, a través de la aplicación de sustancias químicas sintéticas, puede ser una alternativa de control de enfermedades en diferentes cultivos, en los cuales, desencadenan señales que activan varias respuestas de defensa de las plantas (Kessmann et al. 1994; Sticher et al. 1997; Vallad \& Goodman, 2004). Al respecto, compuestos conocidos como "fosfitos", derivados del ácido fosforoso y combinados con otros elementos, como calcio, manganeso, magnesio, potasio o zinc y utilizados normalmente como fertilizantes foliares, han demostrado que promueven el crecimiento y estimulan las plantas, al activar mecanismos de defensa contra enfermedades, como las sigatokas negra y amarilla, en plátano Dominico Hartón (Mogollón \& Castaño, 2011) y el tizón tardío, causado por Phytophthora infestans, en cultivos de papa (Johnson et al. 2004).

Los inductores químicos disponibles comercialmente más estudiados hasta ahora son el benzothiadiazol y el ácido 2,6-dicloroisonicotinico. La inducción sistémica de resistencia con estas dos moléculas ha sido registrada en varias in- teracciones planta-patógeno, que presentaron reducciones entre el 32 y $99 \%$ en el área bajo la curva de progreso de la enfermedad, como por ejemplo, el uso de benzothiadiazol contra Peronosclerospora sorghii, causante del mildeo velloso del maíz; Pseudomonas syringae pv. Tomato, causante de la peca bacterial del tomat; Tomato spotted wilt tospovirus, causante del bronceamiento del tomate INA, para el control de Uromyces appendiculatus, causante de la roya del frijol y Sclerotinia sclerotiorum, causante del moho blanco de la soya (Vallad \& Goodman, 2004).

Estudios citológicos demuestran que el efecto de protección ejercido por benzothiadiazol, se puede explicar por la reducción de la colonización fungosa de los tejidos, asociada con la acumulación masiva de gránulos de almidón en la pared celular, que actúan como barreras estructurales (Görlach et al. 1996). Este reforzamiento mediado por la deposición temprana de calosa y fenoles, probablemente, conduce a cambios, tanto en la rigidez como en la vulnerabilidad de las células y tejidos (Patiño, 2008). Görlach et al. (1996) determinaron que la tasa de penetración de Erysiphe graminis f. sp. tritici en tejido tratado con benzothiadiazol disminuyó en un $30 \%$, debido, posiblemente, a la formación de papilas efectivas en el tejido tratado, que retardan la penetración del hongo.

El acibenzolar-s-metil (ASM), formulado especialmente para ser aplicado en emulsión de aceite-agua, es aplicado como complemento de fungicidas para el control de $M$. fijiensis y M. musicola a intervalos de 40 días. El ASM es absorbido por raíces, por hojas y por tallos y distribuido rápidamente por el xilema y el floema (Thomson, 2011). Según estudios realizados en plátano Dominico Hartón, el ASM aplicado a los cormos en inmersión (dosis de 0,005 $\mathrm{mL} \mathrm{L}^{-1}$ ), antes de siembra, indujo resistencia a las Sigatokas hasta los dos meses de edad de las plántulas (Márquez \& Castaño, 2007). Patiño (2002) registró un aumento entre 30,4 y $22,2 \%$ en el control de M. fijiensis en banano aplicado, en dosis de $40 \mathrm{y}$ $15 \mathrm{~g} \mathrm{ha}^{-1}$, respectivamente.

El fungicida más utilizado en el mundo para el manejo de las Sigatokas es el propiconazole, que previene y controla los patógenos, ya que detiene su desarrollo e interfiere la síntesis del ergosterol en la membrana celular del patógeno. Este fungicida actúa en el momento de la penetración del hongo y, principalmente, en la formación de haustorios secundarios dentro de los tejidos de la planta (Thomson, 2011); sin embargo, su acción no ha sido comparada con la producida por los inductores.

El fosfito de potasio ha sido utilizado para el control, principalmente, de Oomycetes (Garbelotto et al. 2007) y, en menor grado, de nematodos (Salgado et al. 2007). Además de ejercer una acción directa sobre el patógeno interviene en 
el metabolismo de la planta, mediante cambios hormonales y químicos, que incrementan el llenado de frutos y el rendimiento en cultivos de aguacate y de cítricos (Lovatt, 1999). Para el manejo de las Sigatokas negra y amarilla, se evaluó el fosfito de potasio en plántulas de plátano Dominico Hartón y se observó la reducción del grado de evolución de los síntomas y del índice de severidad, con resultados similares al fungicida propiconazole; sin embargo, el inductor y el fungicida actuaron sobre sistemas diferentes, el primero sobre el sistema de defensa de las plántulas y, el segundo, directamente en el patógeno (Mogollón \& Castaño, 2011).

Esta investigación tuvo como objetivo evaluar el efecto de los inductores de resistencia ASM y fosfito de potasio (FP), sólo o en mezcla con propiconazole, para el manejo de las Sigatokas negra y amarilla en plátano Dominico Hartón (Musa AAB Simmonds); además de cuantificar el efecto sobre la producción del cultivo y el impacto económico de los tratamientos.

\section{MATERIALES Y MÉTODOS}

El estudio, se llevó a cabo en la granja 'Montelindo' de la Universidad de Caldas, ubicada en la vereda Santágueda, municipio de Palestina (Caldas), a $5^{\circ} 05^{\prime} \mathrm{N}$ y $75^{\circ} 40^{\prime} \mathrm{W}$ y una altitud de $1.010 \mathrm{~m}$, con precipitación anual de $2.100 \mathrm{~mm}$, humedad relativa promedio de $76 \%$ y temperatura promedio de $23,5^{\circ} \mathrm{C}$,suelos de textura franco arenosa, ligeramente ácidos, de origen volcánico de la clase Typic Distrandept (Cardona et al. 2007).

Se sembraron cormos de plátano provenientes de la misma granja (500g de peso promedio), de la cultivariedad Dominico Hartón, a $3 \mathrm{~m}$ entre surcos y $2 \mathrm{~m}$ entre plantas, previamente tratados, mediante inmersión en carbofuran, como tratamiento preventivo para nematodos y picudos, en dosis de $10 \mathrm{~mL} \mathrm{~L}^{-1}$ de agua, durante una hora. Los surcos fueron separados cada uno por una barrera de plantas de la misma cultivariedad sembradas a $1 \mathrm{~m}$, para contrarrestar el efecto de deriva durante las aplicaciones de los tratamientos, que se realizaron con una aspersora de espalda marca Agrolaura (®) de $20 \mathrm{~L}$ de capacidad, con boquilla de cono a bajo volumen.

Al cultivo se le hizo el adecuado y oportuno manejo agronómico, recomendado por Aranzazu et al. (2002) y, el cual, consistió en las siguientes prácticas: Manejo de arvenses: después de sembrados los cormos se integraron métodos químicos y mecánicos, mediante el uso de guadaña y de glifosato en dosis de 1,5L ha ${ }^{-1}$ a los 3, 6 y 9 meses después de la siembra. Fertilización: se realizaron cinco aplicaciones de fertilizantes, teniendo en cuenta los requerimientos de nutrientes del cultivo de plátano (Martínez, 1997), de la siguiente manera: la primera, al momento de la siembra, con urea $\left(25 \mathrm{~g} \mathrm{sitio}^{-1}\right)$ y boro $\left(10 \mathrm{~g} \mathrm{sitio}^{-1}\right)$; con urea $\left(50 \mathrm{~g} \mathrm{planta}^{-1}\right)$, fósforo $\left(50 \mathrm{~g} \mathrm{planta}^{-1}\right)$, cloruro de potasio $\left(60 \mathrm{~g}_{\text {planta }}{ }^{-1}\right)$ y elementos menores ( $\left.30 \mathrm{~g}_{\text {planta }}{ }^{-1}\right)$; la tercera, con urea $(50 \mathrm{~g}$ planta $\left.^{-1}\right)$, cloruro de potasio $\left(100 \mathrm{~g} \mathrm{planta}^{-1}\right)$ y elementos menores (30 $\left.\mathrm{g}_{\text {planta }} \mathrm{a}^{-1}\right)$; para la cuarta, urea $\left(50 \mathrm{~g} \mathrm{planta}^{-1}\right)$, fósforo $\left(50 \mathrm{~g} \mathrm{planta}^{-1}\right)$, cloruro de potasio $\left(100 \mathrm{~g} \mathrm{planta}^{-1}\right)$ y elementos menores ( $\left.30 \mathrm{~g} \mathrm{planta}^{-1}\right) \mathrm{y}$, finalmente, una quinta, dirigida al hijo mayor, con urea $\left(50 \mathrm{~g}_{\text {planta }}{ }^{-1}\right)$, cloruro de potasio $\left(40 \mathrm{~g} \mathrm{planta}^{-1}\right)$ y elementos menores $\left(30 \mathrm{~g}_{\text {planta }} \mathrm{g}^{-1}\right)$, las cuales, se realizaron a los 3, 6, 912 meses después de la siembra. Deshojes: se realizaron con machete o media luna, según la altura de las plantas, cada 15 días, en los meses lluviosos y mensualmente en los meses secos. Desguasque: consistió en retirar las calcetas viejas y se realizó trimestralmente. Desbellote y desmane: ambas prácticas se realizaron después de la emergencia de la bellota, aproximadamente, a los once meses de la siembra del cultivo; el desmane, se efectuó para dejar cinco manos por racimo.

Se usó un diseño de bloques completos al azar, con ocho tratamientos, cuatro repeticiones y ocho plantas por repetición. Los tratamientos fueron: 1) Acibenzolar-s-metil (40mL $\mathrm{ha}^{-1}$, mitad de dosis comercial); 2) Acibenzolar-s-metil ( $80 \mathrm{~mL}$ $\mathrm{ha}^{-1}$, dosis comercial); 3) Fosfito de potasio (300mL ha $\left.{ }^{1}\right)$; 4) Fosfito de potasio (500 $\mathrm{mL} \mathrm{ha}^{-1}$, dosis comercial); 5) Propiconazole(400 $\mathrm{mL} \mathrm{ha}^{-1}$, dosis comercial); 6) Acibenzolars-metil + Propiconazole (40 y $200 \mathrm{~mL} \mathrm{ha}^{-1}$, respectivamente); 7) Fosfito de potasio + Propiconazole (300 y $200 \mathrm{~mL} \mathrm{ha}^{-1}$, respectivamente) y, 8 ) Testigo (sin aplicación de productos y con deshoje fitosanitario).

Siguiendo las recomendaciones de Syngenta (Thomson, 2011), los inductores de resistencia se aplicaron cada seis semanas en ambas dosis, a partir de los tres meses después de la siembra, cuando las plántulas tenían las primeras hojas verdaderas bien desarrolladas, para un total de siete aplicaciones, durante el ciclo del cultivo. La aplicación de propiconazole sólo y en mezcla con ASM o FP, se realizó de acuerdo con el sistema de preaviso biológico (Aranzazu et al. 2002), consistente en la evaluación semanal del estado de desarrollo de las Sigatokas en el envés y extremo de la hoja No. 4, en diez plantas seleccionadas, desde su estado juvenil (aproximadamente, a los tres meses después de la siembra), las cuales, se reemplazaron al momento de la emisión de la bellota.

En la hoja No. 4, se determinó el estado más avanzado de la enfermedad y el grado del síntoma dominante y se calificó con menos (-), cuando se observaron hasta 50 lesiones por hoja y con más (+), cuando superaron las 50 lesiones (Orjeda, 1998). Al sumar los valores calificados en las 10 plantas se obtuvo el nivel de infección de la hoja de la respectiva semana; se hizo la aplicación del fungicida, cuando el nivel de infección de la hoja No. 4 (NIH4) aumentó en 200 unidades o más, con respecto a la lectura de la semana anterior (Gómez \& Castaño, 2001). 
Las variables evaluadas fueron:

Índice de severidad (IS): se hicieron registros semanales del área afectada de las hojas por las Sigatokas, con base en los diagramas de Stover (1971), modificados por Gauhl (1989), ya que en la zona de estudio se tiene establecida la predominancia de la sigatoka negra sobre la amarilla (Torrado \& Castaño, 2008).

Con estos datos, se determinó el índice de severidad semanal en tres plantas por tratamiento y por repetición, con base en la siguiente fórmula:

$$
\mathrm{IS}=\frac{\sum \mathrm{nb}}{(\mathrm{N}-1) \mathrm{T}} \times 100
$$

En la cual:

IS = Índice de severidad

$\mathrm{n}=$ Número de hojas en cada grado

$\mathrm{b}=$ Grado

$N=$ Número de grados empleados en la escala (7)

$\mathrm{T}=$ Número total de hojas evaluadas.

Tasa de desarrollo de la enfermedad_(r): se cuantificó mediante la siguiente fórmula:

$$
\mathrm{r}=\frac{1}{t_{1}-t_{0}}\left(\log _{e} \frac{X_{1}}{1-X_{1}}-\log _{e} \frac{X_{0}}{1-X_{0}}\right)
$$

Donde:

$\mathrm{r}=$ Tasa de desarrollo

$t_{1}=$ Tiempo final

$t_{0}=$ Tiempo inicial

$X_{1}=$ Índice de severidad final

$X_{0}=$ Índice de severidad inicial (Castaño, 2002)

Cuantificaciones de crecimiento: semanalmente, se hicieron registros de la altura y diámetro de las plantas; para tal fin, se tomaron cinco plantas al azar por repetición y se les midió su altura desde la base de la planta hasta el punto donde se forma el ángulo entre la hoja cigarro y la primera hoja expandida. El diámetro, se midió a $1 \mathrm{~m}$ de altura desde la base de la planta, con la ayuda de un calibrador (Aristizábal \& Herrera, 2003).

Rendimiento $\left(\mathrm{t} \mathrm{ha}^{-1}\right)$ : se obtuvo el peso promedio de los racimos extrapolado a 1.660 plantas ha $^{-1}$ para determinar la relación beneficio-costo, a través de la siguiente fórmula:

$$
\text { B/C = IB/CP (Aristizábal, 2007) }
$$

Donde:

B/C: relación beneficio-costo

IB: ingreso bruto

CP: costo de producción
Para este análisis, se cuantificaron los costos derivados del manejo de las sigatokas, así como los costos de las prácticas agronómicas realizadas.

Las variables relacionadas con el crecimiento y la producción de las plantas en los distintos tratamientos y repeticiones fueron sometidas a análisis de varianza; para la separación de promedios de tratamientos, cuando fue necesario, se empleó la prueba de comparación múltiple de Tukey al 95\% de probabilidad. El índice de severidad fue calculado en el período vegetativo, en floración y en producción y los valores obtenidos, se sometieron a análisis de varianza y de prueba de comparación de Tukey. El nivel de infección en la hoja 4 (NIH4), se calculó semanalmente y los valores obtenidos se graficaron en el sistema de coordenadas en comparación con la precipitación acumulada semanalmente y registrada en una estación climática Tipo A, ubicada en un área adyacente al lote experimental. Para todos los análisis estadísticos, se empleó el programa SAS (Statistical Analysis System).

\section{RESULTADOS Y DISCUSIÓN}

Índice de severidad (IS) de las Sigatokas: según el análisis de varianza, solamente el testigo mostró diferencias estadísticamente significativas respecto a los demás tratamientos para el IS (Tabla 1), observándose que el IS más bajo para el período vegetativo se presentó en el tratamiento con FP en dosis de $300 \mathrm{~mL} \mathrm{ha}^{-1}$. Con la aplicación de FP, en ambas dosis, se observó una reducción significativa en el IS de la Sigatokas en comparación con el testigo (Tabla 1), lo que se puede deber a la acción del fósforo en forma de ion fosfito combinado con el potasio, que estimula el crecimiento de la planta al actuar sobre el sistema hormonal y promover los mecanismos de defensa naturales cuando la planta recibe señales a condiciones adversas (Thomson, 2011).

En floración, el mejor tratamiento fue con FP en dosis de $500 \mathrm{~mL} \mathrm{ha}^{-1}$, mientras que para el período reproductivo fue con ASM en dosis de $40 \mathrm{~mL}^{\text {ha-1 }}{ }^{-1}$ (Tabla 1), esto demuestra una influencia positiva de ambos productos sobre el proceso de inducción de resistencia sistémica a las Sigatokas negra y amarilla en plátano Dominico Hartón; estos resultados concuerdan con los obtenidos por Márquez \& Castaño (2007) y Mogollón \& Castaño (2011), quienes evaluaron, en estado de plántula de esta cultivariedad, el efecto de inductores de resistencia sobre ambas Sigatokas, destacándose ASM y FP.

Tasa de desarrollo ( $r$ ) de las Sigatokas: los tratamientos con ASM + propiconazole, propiconazole sólo y FP en dosis de $300 \mathrm{~mL}$ ha $^{-1}$ tuvieron las tasas de desarrollo más bajas, con valores de 0,0032; 0,0056 y 0,0094, respectivamente (Figura 1), lo que concuerda con los resultados obtenidos por Mogollón \& Castaño (2011), que registran la tasa de desa- 
Tabla 1. Efecto de los inductores de resistencia sobre el índice de severidad de las Sigatokas negra y amarilla en diferentes etapas de desarrollo.

\begin{tabular}{|l|c|c|c|c|}
\hline \multicolumn{1}{|c|}{ Tratamientos } & Dosis $\left(\mathbf{m L ~ h a ~}^{-{ }^{-1}}\right)$ & IS PV $^{\mathbf{1}}$ & IS PF $^{2}$ & IS PR $^{3}$ \\
\hline ASM & 40 & $36 a^{*}$ & $22 a$ & $28 a$ \\
\hline ASM & 80 & $35 a$ & $20 a$ & $30 a$ \\
\hline FP & 300 & $32 a$ & $29 a$ & $31 a$ \\
\hline FP & 500 & $42 a$ & $17 a$ & $29 a$ \\
\hline Propiconazole & 400 & $37 a$ & $19 a$ & $30 a$ \\
\hline ASM + propiconazole & $40+200$ & $34 a$ & $25 a$ & $30 a$ \\
\hline FP+ propiconazole & $300+200$ & $43 a$ & $18 a$ & $31 a$ \\
\hline Testigo (deshoje) & - & $46 \mathrm{~b}$ & $34 \mathrm{~b}$ & $48 \mathrm{~b}$ \\
\hline
\end{tabular}

${ }^{1}$ IS PV: índice de severidad, período vegetativo

${ }^{2}$ IS PF: índice de severidad, período de floración

${ }^{3}$ IS PR: índice de severidad, período reproductivo

"Promedios acompañados de letras diferentes denotan diferencias estadísticas significativas, según el rango de comparación múltiple de Tukey al 5\% de probabilidad.

rrollo más baja con FP en dosis de $27 \mathrm{~mL} \mathrm{~L}^{-1}$ sin diferencia con propiconazole.

Por otro lado, en un estudio realizado por Márquez \& Castaño (2007), la tasa de desarrollo más baja se obtuvo con ASM en dosis de $0,03 \mathrm{~mL} \mathrm{~L}^{-1}$, el cual, presentó diferencias significativas con propiconazole. Esto indica que el resultado de la aplicación de los inductores sólo o en mezcla con el fungicida fue similar al del manejo tradicional. Estos resultados también coinciden con lo recomendado para la aplicación de ASM en mezcla con un fungicida (Thomson, 2011).

Con base en el NIH4 y la precipitación acumulada durante el período de evaluación, fue necesario realizar seis aplicaciones de propiconazole sólo y en mezcla con ASM y FP (Figura 2), igual a lo reportado para la misma zona de estudio y cultivariedad, por Aristizábal et al. (2006). Se puede observar en la figura 2, que las aplicaciones se hicieron después de presentarse las máximas precipitaciones, con el fin de reducir el nivel de infección de las Sigatokas en la hoja $N^{\circ} 4$, que bajó entre 200 y 350 unidades. Resultados similares fueron reportados por Ramírez \& Rodríguez (1996) y Pérez (1998), quienes indican que la mayor severidad de las Sigatokas está relacionada con las más altas precipitaciones, las cuales, en la vereda de Santágueda, corresponden a los meses de marzo a mayo y septiembre a noviembre.

Además de la ventaja socioeconómica y ambiental de utilizar el nivel de infección en la hoja cuatro (NIH4) para el manejo de las Sigatokas, se disminuyen los riesgos de una posible resistencia desarrollada por sus agentes causantes, en par- ticular a productos de acción sistémica, ya que se reducen en un tercio las aplicaciones de fungicidas (Cardona et al. 2007).

Variables de crecimiento: no hubo diferencias estadísticamente significativas entre tratamientos respecto a las variables altura de planta y diámetro del pseudotallo (datos no mostrados). Según Aristizábal et al. (2006), los tratamientos aplicados contra las Sigatokas no tienen efecto en el crecimiento de la planta, puesto que únicamente es afectado cuando no se realiza un plan de fertilización balanceado.

Los períodos a belloteo y a cosecha variaron de 39 a 41 y de 50 a 53 semanas después de siembra, respectivamente (datos no mostrados), inferiores a los reportados por Aristizábal \& Herrera (2003), en la misma región. En frutos de naranja afectados por Penicillium digitatum, se estableció que, una vez finalizado el tratamiento con inductores de resistencia, se produce un aumento en la síntesis de etileno en los frutos; en el mismo sentido, Fan et al. (1997) afirman que el aumento en los niveles de etileno en la planta se produce en respuesta a situaciones de estrés, como el daño mecano, por ejemplo, pero también al ataque de organismos patógenos, de otra parte; se sabe que el etileno induce fenómenos de senescencia que pueden acortar las fenofases de un cultivo y, en consecuencia, reducir el ciclo de vida de la planta (Yang et al. 2008).

Análisis económico: en términos de rendimiento, con una población de 1.666 plantas ha ${ }^{-1}$, los mejores tratamientos fueron ASM en ambas dosis y en mezcla con propiconazole 

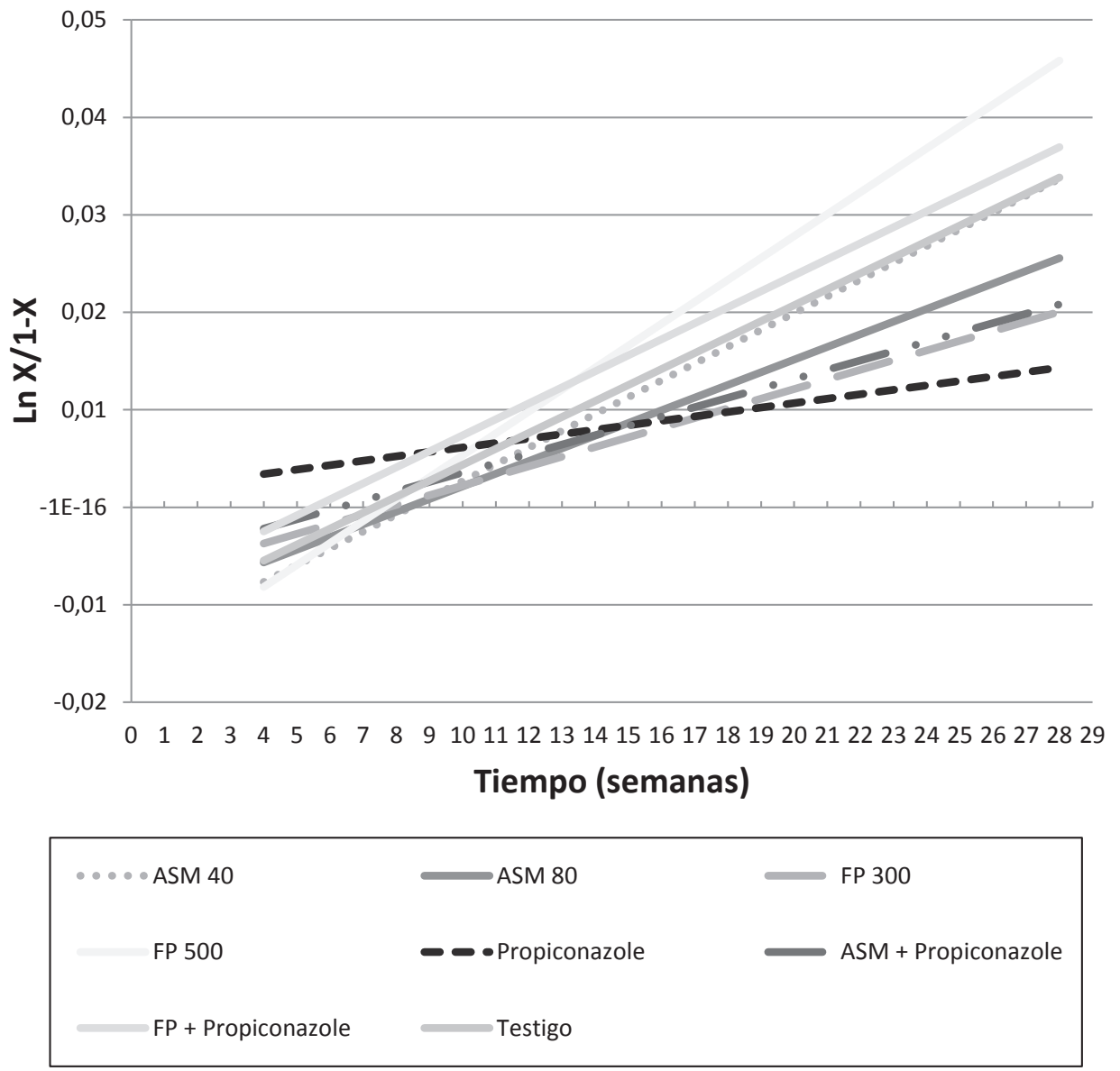

Figura 1. Tasas de desarrollo de las Sigatokas negra y amarilla, a través del tiempo, en plátano Dominico Hartón, de acuerdo con los tratamientos.

y FP, los cuales, aumentaron el rendimiento en 6,0, 3,3, 5,4 y 3,0 ton $\mathrm{ha}^{-1}$, con respecto al testigo, respectivamente (Tabla 2).

El empleo de inductores de resistencia produjo beneficios económicos en el manejo de las Sigatokas negra y amarilla. El tratamiento con mejor relación B/C fue ASM en dosis de $80 \mathrm{~mL} \mathrm{ha}^{-1}$, seguido de los tratamientos con ASM en dosis de $40 \mathrm{~mL} \mathrm{ha}^{-1}$ y la mezcla FP + propiconazole. Se evidencia la ventaja de los inductores de resistencia respecto a propiconazole, aunque los costos de producción fueron similares; los ingresos netos para ASM en ambas dosis fueron superiores a los de propiconazole, en 1,2 y 1,5 veces y a los del testigo en 1,3 y 1,7 veces, respectivamente. Además, la relación beneficio-costo, en ambas dosis, fue de 1,2 y 1,5 por encima del testigo, respectivamente (Tabla 2).

La viabilidad de la utilización de las alternativas de manejo de las Sigatokas negra y amarilla con inductores de resistencia, como modelo de adopción de tecnología, se puede deducir de la relación $\mathrm{B} / \mathrm{C}$ (Tabla 2 ), que también es una alternati- va económica y ambientalmente favorable para el manejo de estas enfermedades, debido a que se disminuye el número de aplicaciones de fungicidas por ciclo de cultivo. En conclusión, los tratamientos con inductores de resistencia sólo o en mezcla con propiconazole para el manejo de los agentes causantes de las Sigatokas negra y amarilla, aunque hayan sido estadísticamente similares al manejo convencional, plantea una solución al problema de la resistencia a los fungicidas, el cual, incrementa el número de aplicaciones por ciclo de cultivo.

Las variables analizadas mostraron un comportamiento comparable, a través del tiempo entre inductores de resistencia y de fungicidas, que permite deducir una influencia positiva del ASM sobre el proceso de resistencia sistémica inducida en el cultivo de plátano.

El conjunto de beneficios incluye, no solamente la reducción en los costos de manejo de las Sigatokas, por el uso de los inductores de resistencia, sino también la reducción de la frecuencia de aplicación de fungicidas por ciclo de cultivo. 


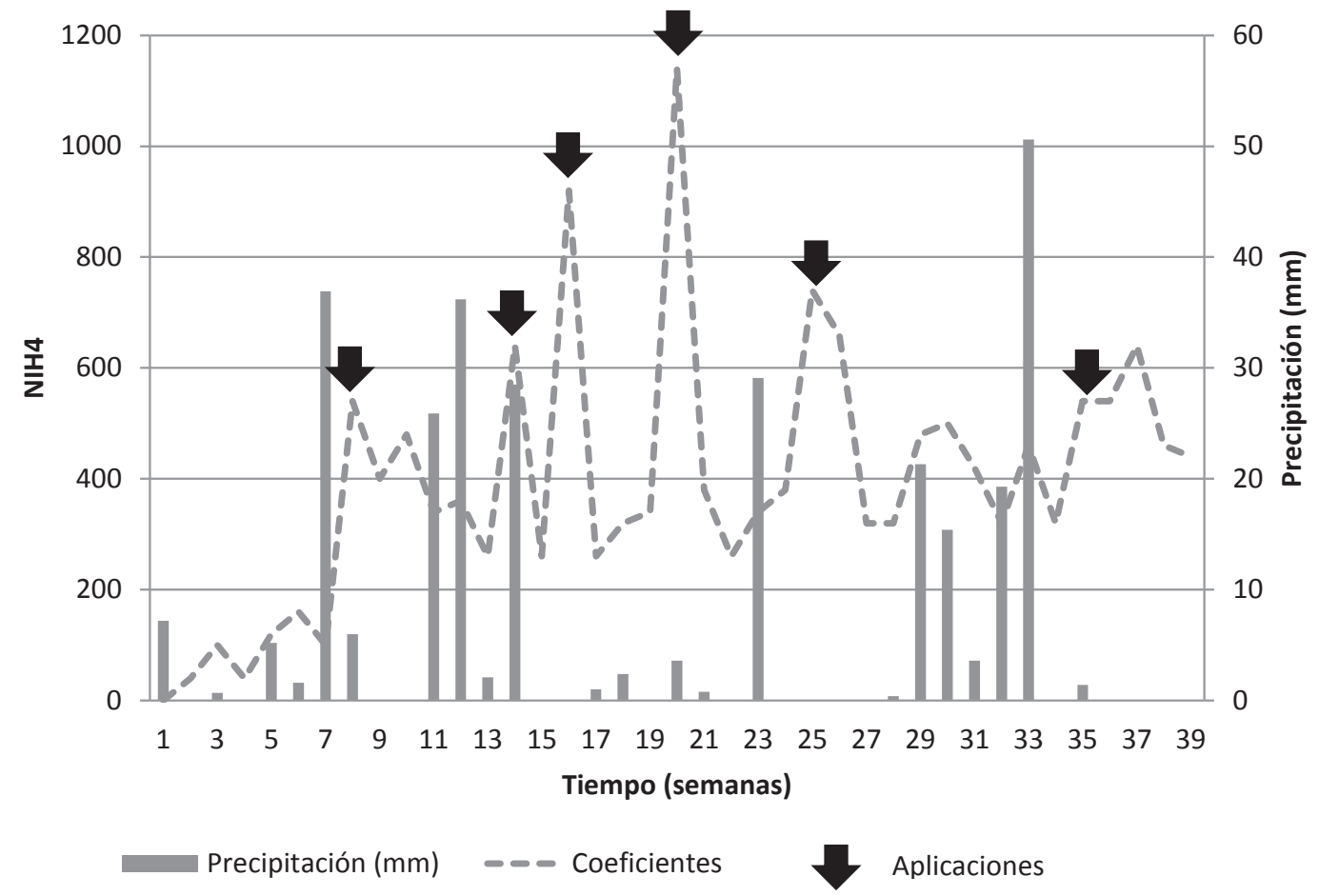

Figura 2. Comportamiento de las Sigatokas negra y amarilla en plátano Dominico Hartón, según el NIH 4 y la precipitación acumulada semanalmente.

Tabla 2. Análisis económico de la aplicación de inductores de resistencia para el manejo de las Sigatokas negra y amarilla en plátano Dominico Hartón.

\begin{tabular}{|c|c|c|c|c|c|c|}
\hline Tratamientos & $\begin{array}{c}\text { Dosis } \\
\left(\mathrm{mL} \mathrm{ha}^{-1}\right)\end{array}$ & $\begin{array}{l}\text { Rendimiento } \\
\left(\mathrm{t} \mathrm{ha}^{-1}\right)\end{array}$ & $\begin{array}{l}\text { Costo de } \\
\text { producción } \\
(\mathrm{kg})\end{array}$ & $\begin{array}{l}\text { Ingreso } \\
\text { bruto }^{1}\end{array}$ & $\begin{array}{l}\text { Ingreso } \\
\text { neto }\end{array}$ & Relación B/C* \\
\hline ASM & 40 & 21 & $5 ’ 654.400$ & $37 ’ 500.000$ & $31^{\prime} 845.600$ & 6,6 \\
\hline ASM & 80 & 18,3 & $6 ’ 779.400$ & $46 ’ 800.000$ & $40 ’ 020.600$ & 6,9 \\
\hline FP & 300 & 15 & 3'538.150 & $21^{\prime} 000.000$ & $17^{\prime} 461.850$ & 5,9 \\
\hline FP & 500 & 18 & $4 ' 546.900$ & $22 ' 800.000$ & $18 ' 253.100$ & 5,0 \\
\hline Propiconazole & 400 & 17,3 & 5’704.000 & $33^{\prime} 150.000$ & $27^{\prime} 446.000$ & 5,8 \\
\hline ASM + propiconazole & $40+200$ & 20,4 & 6'954.000 & $32 ' 400.000$ & $25^{\prime} 446.000$ & 4,6 \\
\hline FP+ propiconazole & $300+200$ & 17,1 & 4’721.500 & $31 ’ 200.000$ & $26 ' 478.500$ & 6,6 \\
\hline Testigo (deshoje) & - & 15 & 4'329.400 & $28,050.000$ & $23^{\prime} 720.600$ & 5,4 \\
\hline
\end{tabular}

${ }^{1}$ El precio de venta fue de $\$ 1.500 \mathrm{~kg}^{-1}$ en el punto de venta de la Universidad de Caldas.

*B/C: relación beneficio-costo 
Agradecimientos: A la empresa Magro, por el suministro de los inductores de resistencia y a la Vicerrectoría de Investigaciones y Postgrados de la Universidad de Caldas, por el apoyo financiero. Conflictos de intereses: El manuscrito fue preparado y revisado con la participación de todos los autores, quienes declaramos que no existe ningún conflicto de intereses que ponga en riesgo la validez de los resultados presentados. Financiación: Este estudio fue financiado por la Vicerrectoría de Investigaciones y Postgrados de la Universidad de Caldas.

\section{BIBLIOGRAFÍA}

1. AGRONET, 2012. Reportes estadísticos, producción nacional por producto. Ministerio de Agricultura y Desarrollo Rural. Colombia. Disponible desde internet en: http://www.agronet.gov.co/agronetweb1/ Estad\%c3\%adsticas/ReportesEstad\%c3\%adsticos. aspx (con acceso 02/28/2012).

2. ARANZAZU, F.; VALENCIA, J.; ARCILA, M.I.; CASTRILLÓN, C.; BOLAÑOS, M.M.; CASTELLANOS, P.A.; PÉREZ, J.C.; RODRÍGUEZ, J.L. 2002. El Cultivo de plátano. En: Manual Técnico. Manizales: CORPOICA. p.80-82.

3. ARISTIZÁBAL, L.M. 2007. Impacto económico de la práctica del desmane en el híbrido de plátano FHIA20. Boletín Fitotecnia.127. Universidad de Caldas. Manizales. 2p.

4. ARISTIZÁBAL, L.M.; HERRERA, J.W. 2003. Características agronómicas de híbridos y cultivariedades de plátano en la región de Santágueda. Boletín Fitotecnia. 082.2p.

5. ARISTIZÁBAL, L.M.; OROZCO, P.M.L.; OSTOS, A.M.A. 2006. Efectos del sistema de manejo de las Sigatokas y la frecuencia de fertilización sobre el crecimiento y producción del plátano Dominico Hartón (Musa AAB). Rev. Cie. Agr. 23(1-2):74-93.

6. ARZANLOU, M.; GROENEWALD, J.Z.; FULLERTON, R.A.; ABELN, E.C.A.; CARLIE, J.; ZAPATER, M.F.; BUDDENHAGEN, I.W.; VILJOEN, A.; CROUS, P.W. 2008. Multiple gene genealogies and phenotypic characters differentiate several novel species of Mycosphaerella and related anamorphs on banana. Persoonia. 20:19-37.

7. CASTAÑO, J. 2002. Principios básicos de fitoepidemiología. Universidad de Caldas, Manizales. 398p.

8. CARDONA, S.; LONDOÑO, S.; CASTAÑO, J.; ARISTI-
ZABAL, M. 2007. Evaluación de métodos de manejo de las Sigatokas amarilla (Mycosphaerella musicola Leach) y negra (Mycosphaerella fijiensis Morelet) del plátano (Musa AAB). Fitopat. Col. 31(1):23-28.

9. CRAENEN, K.; ORTIZ, R. 2003. Genetic improvement for a sustainable management of resistance. In: Jacome, L.; Lepoivre, P.; Marin, D.; Ortiz, R.; Romero, R.; Escalant, J.V. (eds.). Mycosphaerella leaf spot diseases of bananas: present status and outlook. Proceedings of the 2nd International Workshop on Mycosphaerella leaf spot diseases held in San José, Costa Rica, 20-23 Mayo 2002. INIBAP, Roma. p.181198.

10. CHICA, R.; HERRERA, M.; JIMÉNEZ, I.; LIZCANO, S.; MONTOYA, J.A.; PATIÑO, L.F.; RODRÍGUEZ, P.A.; RUÍZ, L.H. 2004. Impacto y manejo de la Sigatoka negra en el cultivo del banano de exportación en Colombia. En. XVI Reunión Internacional ACORBAT, Oaxaca, México. Memorias. Oaxaca: Acorbat. p.5362.

11. FAN, L.; ZHENG, S.; WANG, X. 1997. Antisense suppression of phospholipase $\mathrm{D} \alpha$ retards abscisic acid and ethylene-promoted senescence of postharvest Arabidopsis leaves. The Plant Cell. 9:2183-2196.

12. FAOSTAT, 2012. Organización de las Naciones Unidas para la Alimentación y la Agricultura, FAO. Disponible desde internet en: http://faostat.fao.org/site/339/ default.aspx (con acceso el 02/28/2012).

13. GARBELOTTO, M.; SCHMIDT, D.J.; HARNIK, T.Y. 2007. Phosphite injections and bark application of Phosphite + Pentrabark $^{\mathrm{TM}}$ control sudden oak death in coast live oak. Arbor. \& Urb. For. 33(5):309-317.

14. GAUHL, F. 1989. Epidemiología y ecología de la Sigatoka negra (Mycosphaerella fijiensis Morelet) en plátano (Musa sp.) en Costa Rica, UPEB. Panamá. 126 p.

15. GÓMEZ, L.A.; CASTAÑO, J. 2001. Manejo integrado de las Sigatokas negra y amarilla en la cultivariedad de plátano África. InfoMusa. 10(2):3-7.

16. GÖRLACH, J.; VOLRATH, S.; KNAUF-BEITER, G.; HENGY, G.; BECKHOVE, U.; KOGEL, K.H.; OOSTENDORP, M.; STAUB, T.; WARD, E.; KESSMANN, H.; RYALS, J. 1996. Benzothiadiazole, a novel class of inducers of systemic acquired resistance, activates gene expression and disease resistance in wheat. The Plant Cell. 8:629-643. 
17. JOHNSON, D.A.; INGLIS, D.A.; MILLER, J.S. 2004. Control of potato tuber rots caused by oomycetes with foliar applications of phosphorous acid. Plant Dis. 88:1153-1159.

18. KESSMANN, H.; STAUB, T.; HOFMAN, C.; MAETZKE, T.; HERZOG, J.; WARD, E.; UKNES, S.; RYALS, J. 1994. Induction of systemic acquired disease resistance in plants by chemicals. Ann. Rev. Phytopath. 32:439-459.

19. LOVATT, C.J. 1999. Timing citrus and avocado foliar nutrient applications to increase fruit set and size. Hort. Tech. 9:607-612.

20. MÁRQUEZ, L.J.; CASTAÑO, J. 2007. Inducción de resistencia a Sigatokas en plántulas de plátano Dominico Hartón. Agronomía 15(2):49-57.

21. MARTÍNEZ, G.A. 1997. Mineral nutrient deficiency in plantain. Hohenheim Tropical. Agricultural Series. Margraf Verlag, Weikersheim, Germany. 112p.

22. MOGOLLÓN, A.M.; CASTAÑO, J. 2011. Efecto de inductores de resistencia en plántulas de plátano Dominico Hartón (Musa balbisiana AAB) contra Mycosphaerella spp. Rev. Acad. Col. Cienc. 35(137):463-471.

23. ORJEDA, G. 1998. Evaluación de la resistencia de los bananos a las enfermedades de Sigatoka y Marchitamiento por Fusarium. En: Guías técnicas INIBAP 3. Inst. Int. Recursos Fitogenéticos, Roma, Italia; Red Int. para el Mejoramiento del Banano y el Plátano, Montpellier, Francia. 63p.

24. PATIÑO, L.F. 2002. Efecto de una fuente de energía, tres inductores de resistencia y un sustrato foliar sobre Sigatoka negra en banano. En: Memorias XV Reunión Internacional ACORBAT (Cartagena de Indias, Colombia). Medellín: Augura. p.135-142.

25. PATIÑO, L.F. 2008. Resistencia inducida mediante sustancias químicas a enfermedades causadas por hongos en plantas. Fitopat. Col. 32(1):25-30.

26. PÉREZ, L. 1998. Control de Sigatoka negra en Cuba: Un enfoque de manejo integrado de la enfermedad. InfoMusa. 7(1):26-28.
27. RAMÍREZ, S.G.; RODRÍGUEZ, C.J. 1996. Manual de producción de plátano para Tabasco y Norte de Chiapas. INIFAP. CIRGOC. Campo Experimental Huimanguillo. Tabasco, México. Folleto Técnico No. 13. 80p.

28. RIVEROS, A.S. 2001. Moléculas activadoras de la resistencia inducida, incorporadas en programas de Agricultura Sostenible. Rev. Manejo Integrado de Plagas (Costa Rica). 61:4-11.

29. SALGADO, S.M.; RESENDE, M.L.; CAMPOS, V.P. 2007. Efeito de indutores de resistência sobre Meloidogyne exigua do cafeeiro. Ciênc. Agrotec. 31(4):10071013.

30. STICHER, L.; MAUCH-MANI, B.; MÉTRAUX, J.P. 1997. Systemic acquired resistance. Ann. Rev. Phytopath. 35:235-270.

31. STOVER, R.H. 1971. A proposed international scale for estimating intensity of banana leaf spot (Mycosphaerella musicola Leach).Trop. Agr. (Trinidad). 48(3):185-196.

32. THOMSON PLM. 2011. Diccionario de especialidades agroquímicas. Colombia. Edición №21.

33. TORRADO, M.; CASTAÑO, J. 2008. Incidencia y severidad de las sigatokas negra (Mycosphaerella fijiensis Morelet) y amarilla (Mycosphaerella musicola Leach et Mulder) del plátano según los estados fenológicos. Agr. Col. 26(3):435-442.

34. VALLAD, G.E.; GOODMAN, R.M. 2004. Systemic acquired resistance and induced systemic resistance in conventional agriculture. Crop Sci. 44:1920-1934.

35. YANG, T.F.; CONZALEZ-CARRANZA, Z.H.; MAUNDERS, M.J.; ROBERTS, A.J. 2008. Ethylene and the regulation of senescence processes in transgenic Nicotiana sylvestris plants. Ann. Bot. 101(2):301-310.

Recibido: Mayo 17 de 2012

Aceptado: Septiembre 28 de 2012 